\title{
FIXED POINT RESULTS OF RATIONAL TYPE CONTRACTIONS IN $b$-METRIC SPACES
}

\author{
MIAN BAHADUR ZADA ${ }^{1}$, MUHAMMAD SARWAR ${ }^{1, *}$ AND POOM KUMAM ${ }^{2, *}$ \\ ${ }^{1}$ Department of Mathematics University of Malakand, Khyber Pakhtunkhwa, Chakdara Dir(L), Pakistan \\ ${ }^{2}$ Department of Mathematics, Faculty of Science, King Mongkut's University of Technology Thonburi \\ (KMUTT), 126 Pracha Uthit Road, Bang Mod, Thung Khru, Bangkok 10140, Thailand \\ *Corresponding authors: sarwar@uom.edu.pk, poom.kum@kmutt.ac.th
}

\begin{abstract}
The aim of this manuscript is to establish fixed point results satisfying contractive conditions of rational type in the setting of $b$-metric spaces. The results proved herein are the generalization and extension of some well known results in the existing literature. Example is also given in order to illustrate the validity of the presented results.
\end{abstract}

\section{Introduction And Preliminaries}

The Banach contraction principle [2] is considered to be the pioneering result of the fixed point theory, and plays an important role for solving existence problems in many branches of nonlinear analysis. This principle asserts if $(X, d)$ is a complete metric space and $K: X \rightarrow X$ satisfies

$$
d(K x, K y) \leq \lambda d(x, y)
$$

for all $x, y \in X$ with $\lambda \in[0,1)$, then $K$ has a unique fixed point.

This principle have been improved and extended by several mathematicians in different directions some of them are as follows: Let $K$ be a mapping on a metric space $(X, d)$ and $x, y \in X$, then $K$ is said to be

Received 2018-06-11; accepted 2018-08-13; published 2018-11-02.

2010 Mathematics Subject Classification. Primary 47H10; Secondary 54H25.

Key words and phrases. $b$-metric spaces; common fixed points; self-maps; Cauchy sequence; contractive conditions.

(C)2018 Authors retain the copyrights of their papers, and all open access articles are distributed under the terms of the Creative Commons Attribution License. 
(1) Kannan type contraction [10], if there exists a number $\lambda \in\left[0, \frac{1}{2}\right)$ such that

$$
d(K x, K y) \leq \lambda[d(x, K x)+d(y, K y)]
$$

(2) Chatterjee type contraction [4], if there exists a number $\lambda \in\left[0, \frac{1}{2}\right)$ such that

$$
d(K x, K y) \leq \lambda[d(x, K y)+d(y, K x)]
$$

(3) Reich type contraction [12], if there exists a number $\lambda, \mu, \nu \in[0,1)$ with $\lambda+\mu+\nu<1$ such that

$$
d(K x, K y) \leq \lambda d(x, y)+\mu d(x, K x)+\nu d(y, K y) .
$$

(4) Das and Gupta [7] rational type contraction, if there exists a number $\lambda, \mu \in[0,1)$ with $\lambda+\mu<1$ such that

$$
d(K x, K y) \leq \lambda d(x, y)+\mu \frac{d(y, K y)[1+d(x, K x)]}{1+d(x, y)} .
$$

The contractive conditions on underlying functions play an important role for finding solutions of metric fixed point problems. Inspired from the impact of this natural idea, the above contractions have been extended and generalized by several researchers in various spaces such as quasi-metric spaces, cone metric spaces, G-metric spaces, partial metric spaces and vector valued metric spaces etc. Following this trend, Bakhtin [1] and Czerwik [5] generalized metric space with non Hausdorff topology called $b$-metric space to overcome the problem of measurable functions with respect to measure and their convergence. They presented the generalization of the Banach contraction principle in $b$-metric spaces. Since then, several papers has been studied by many authors dealing with the existence of fixed point in $b$-metric spaces (see, $[3,6,8,9,11,13,14]$ and the references therein).

The aim of this contribution is to investigate some fixed point results using the concept of the contractive conditions of rational type in the context of $b$-metric spaces. Moreover, an example is given here to illustrate the validity of the obtained results. Actually the derived results generalizes the results of $[2,4,7,10,12]$.

Now, we recall some essential notations, definitions and primary results known in the literature. Throughout this manuscript, $\mathbb{R}=$ set of real numbers, $\mathbb{R}^{+}=[0, \infty)$ and $\mathbb{N}=$ set of positive integers.

Definition 1.1. [1,5] Let $X$ be a nonempty set. A function $d: X \times X \rightarrow \mathbb{R}^{+}$is called a $b$-metric with coefficient $s \geq 1$ if:

(1) $d(x, y)=0 \Leftrightarrow x=y$

(2) $d(x, y)=d(y, x) \quad \forall x, y \in X$;

(3) $d(x, y) \leq s[d(x, y)+d(z, y)] \quad \forall x, y, z \in X$.

Then the pair $(X, d)$ is called a b-metric space. 
Remark 1.1. Every metric space is b-metric space with $s=1$, but in general, a b-metric space need not necessarily be a metric space, as in below example 1.1, $(X, d)$ is b-metric space but not a metric space (see also, examples in [6,13]). Thus, the class of b-metric spaces is larger than the class of metric spaces.

Example 1.1. Let $X=\mathbb{R}$ and let the mapping $d: X \times X \rightarrow \mathbb{R}^{+}$be defined by $d(x, y)=|x-y|^{2}$ for all $x, y \in X$. Then $(X, d)$ is a $b-$ metric space with coefficient $s=2$.

Sintunavarat [14] generalized Example 1.1 as:

Example 1.2. Let $(X, \rho)$ be a metric space and $p \geq 1$ be a given real number. Then $d(x, y)=[\rho(x, y)]^{p}$ is a b-metric on $X$ with parameter $s \leq 2^{p-1}$.

The following example 1.3 shows that $b$-metric is not continuous in general (see also, examples in [9,11]).

Example 1.3. [8] Let $X=\mathbb{N} \cup\{\infty\}$ and $d: X \times X \rightarrow \mathbb{R}$ be defined by

$$
d(m, n)= \begin{cases}0, & \text { if } m=n, \\ \left|\frac{1}{m}+\frac{1}{n}\right|, & \text { if one of } m, n \text { is even and the other is even or } \infty, \\ 5, & \text { if one of } m, n \text { is odd and the other is odd (and } m=n \text { ) or } \infty, \\ 2, & \text { otherwise. }\end{cases}
$$

Then, considering all possible cases, it can be checked that $(X, d)$ is a b-metric space with $s=\frac{5}{2}$. However, let $x_{n}=2 n$ for each $n \in \mathbb{N}$. Then $\lim _{n \rightarrow \infty} d(2 n, \infty)=\lim _{n \rightarrow \infty} \frac{1}{2 n}=0$, that is, $x_{n} \rightarrow \infty$, but $d\left(x_{n}, 1\right)=2 \nrightarrow 5=d(\infty, 1)$ as $n \rightarrow \infty$.

Definition 1.2. [3] Let $\left\{x_{n}\right\}$ be a sequence in b-metric space $(X, d)$ and $x \in X$. Then

(1) $\left\{x_{n}\right\}$ converges to $x$ if and only if for every $\varepsilon>0$, there exists $n(\varepsilon) \in \mathbb{N}$, such that $d\left(x_{n}, x\right)<\varepsilon$ for all $n>n(\varepsilon)$ and we write $\lim _{n \rightarrow \infty} d\left(x_{n}, x\right)=0$ or $\lim _{n \rightarrow \infty} x_{n}=x$.

(2) $\left\{x_{n}\right\}$ is a Cauchy sequence if for every $\varepsilon>0$, there exists $n(\varepsilon) \in \mathbb{N}$, such that $d\left(x_{n}, x_{m}\right)<\varepsilon$ for all $m, n>n(\varepsilon)$.

Proposition 1.1. [3] In a b-metric space $(X, d)$, the following assertions hold:

- a convergent sequence has a unique limit;

- each convergent sequence is Cauchy;

- a metric space $(X, d)$ is complete if every Cauchy sequence is convergent in $X$.

\section{FiXed point Results in $b$-Metric spaCes}

To present the main results, we need the following lemma. 
Lemma 2.1. Let $(X, d)$ be a complete b-metric space and $L: X \rightarrow X$. Let $x_{0} \in X$ and define the sequence $\left\{x_{n}\right\}$ by

$$
L x_{n}=x_{n+1} \quad \forall \quad n=0,1,2, \ldots
$$

Let there exists a mapping $\lambda: X \times X \rightarrow[0,1)$ satisfying

$$
\lambda(L x, y) \leq \lambda(x, y) \quad \text { and } \quad \lambda(x, L y) \leq \lambda(x, y), \text { for all } \quad x, y \in X .
$$

Then

$$
\lambda\left(x_{n}, y\right) \leq \lambda\left(x_{0}, y\right) \text { and } \lambda\left(x, x_{n+1}\right) \leq \lambda\left(x, x_{1}\right) \text { for all } x, y \in X \text { and } n=0,1,2, \ldots
$$

Proof. Let $x, y \in X$ and $n=0,1,2, \ldots$, then

$$
\lambda\left(x_{n}, y\right)=\lambda\left(L x_{n-1}, y\right) \leq \lambda\left(x_{n-1}, y\right)=\lambda\left(L x_{n-2}, y\right) \leq \lambda\left(x_{n-2}, y\right)=\ldots \leq \lambda\left(x_{0}, y\right) .
$$

Similarly,

$$
\left.\lambda\left(x, x_{n+1}\right)=\lambda\left(x, L x_{n}\right) \leq \lambda\left(x, x_{n}\right)=\lambda\left(x, L x_{n-1}\right)\right)=\ldots \leq \lambda\left(x, x_{0}\right) .
$$

Now, we prove the main result.

Theorem 2.1. Let $(X, d)$ be a complete $b$-metric space and $\lambda_{i}: X \times X \rightarrow[0,1), i=1,2, \ldots, 6$. If $L: X \rightarrow X$ be a self-map such that for all $x, y \in X$ the following conditions are satisfied:

(i) $\lambda_{i}(L x, y) \leq \lambda_{i}(x, y)$ and $\lambda_{i}(x, L y) \leq \lambda_{i}(x, y)$;

(ii)

$$
\begin{aligned}
d(L x, L y) \leq & \lambda_{1}(x, y) d(x, y)+\lambda_{2}(x, y) \frac{[d(x, L y)+d(y, L x)]}{s} \\
& +\lambda_{3}(x, y)[d(x, L x)+d(y, L y)]+\lambda_{4}(x, y) \frac{d(y, L y)[1+d(x, L x)]}{1+d(x, y)} \\
& +\lambda_{5}(x, y) \frac{d(x, L y) d(x, L x)}{s[1+d(x, y)]}+\lambda_{6}(x, y) \frac{d(x, L y) d(y, L x)}{s[1+d(x, y) d(y, L x)]}
\end{aligned}
$$

where $\lambda_{2}(x, y)+\lambda_{3}(x, y)+\lambda_{5}(x, y)+s \sum_{i=1}^{6} \lambda_{i}(x, y)<1$, with $0 \leq \sum_{i=1}^{6} \lambda_{i}(x, y)<1$. Then the mapping L has a unique fixed point in $X$.

Proof. Let $x_{0} \in X$ and construct a sequence $\left\{x_{n}\right\}$ by the rule

$$
L x_{n}=x_{n+1}, \quad \forall \quad n=0,1,2, \ldots
$$

First, we show that $\left\{x_{n}\right\}$ is a Cauchy sequence in $X$. For this, consider

$$
d\left(x_{n+1}, x_{n+2}\right)=d\left(L x_{n}, L x_{n+1}\right)
$$


by using condition $(i i)$ of Theorem 2.1 with $x=x_{n}$ and $y=x_{n+1}$, we have

$$
\begin{aligned}
d\left(L x_{n}, L x_{n+1}\right) \leq & \lambda_{1}\left(x_{n}, x_{n+1}\right) d\left(x_{n}, x_{n+1}\right)+\lambda_{2}\left(x_{n}, x_{n+1}\right) \frac{\left[d\left(x_{n}, L x_{n+1}\right)+d\left(x_{n+1}, L x_{n}\right)\right]}{s} \\
& +\lambda_{3}\left(x_{n}, x_{n+1}\right)\left[d\left(x_{n}, L x_{n}\right)+d\left(x_{n+1}, L x_{n+1}\right)\right] \\
& +\lambda_{4}\left(x_{n}, x_{n+1}\right) \frac{d\left(x_{n+1}, L x_{n+1}\right)\left[1+d\left(x_{n}, L x_{n}\right)\right]}{1+d\left(x_{n}, x_{n+1}\right)} \\
& +\lambda_{5}\left(x_{n}, x_{n+1}\right) \frac{d\left(x_{n}, L x_{n+1}\right) d\left(x_{n}, L x_{n}\right)}{s\left[1+d\left(x_{n}, x_{n+1}\right)\right]} \\
& +\lambda_{6}\left(x_{n}, x_{n+1}\right) \frac{d\left(x_{n}, L x_{n+1}\right) d\left(x_{n+1}, L x_{n}\right)}{s\left[1+d\left(x_{n}, x_{n+1}\right) d\left(x_{n+1}, L x_{n}\right)\right]}
\end{aligned}
$$

using (2.1), we get

$$
\begin{aligned}
d\left(x_{n+1}, x_{n+2}\right) \leq & \lambda_{1}\left(x_{n}, x_{n+1}\right) d\left(x_{n}, x_{n+1}\right)+\lambda_{2}\left(x_{n}, x_{n+1}\right) \frac{\left[d\left(x_{n}, x_{n+2}\right)+d\left(x_{n+1}, x_{n+1}\right)\right]}{s} \\
& +\lambda_{3}\left(x_{n}, x_{n+1}\right)\left[d\left(x_{n}, x_{n+1}\right)+d\left(x_{n+1}, x_{n+2}\right)\right] \\
& +\lambda_{4}\left(x_{n}, x_{n+1}\right) \frac{d\left(x_{n+1}, x_{n+2}\right)\left[1+d\left(x_{n}, x_{n+1}\right)\right]}{1+d\left(x_{n}, x_{n+1}\right)} \\
& +\lambda_{5}\left(x_{n}, x_{n+1}\right) \frac{d\left(x_{n}, x_{n+2}\right) d\left(x_{n}, x_{n+1}\right)}{s\left[1+d\left(x_{n}, x_{n+1}\right)\right]} \\
& +\lambda_{6}\left(x_{n}, x_{n+1}\right) \frac{d\left(x_{n}, x_{n+2}\right) d\left(x_{n+1}, x_{n+1}\right)}{s\left[1+d\left(x_{n}, x_{n+1}\right) d\left(x_{n+1}, x_{n+1}\right)\right]}
\end{aligned}
$$

with the help of condition $(i)$ of Theorem 2.1, we get

$$
\begin{aligned}
d\left(x_{n+1}, x_{n+2}\right) \leq & \lambda_{1}\left(x_{0}, x_{0}\right) d\left(x_{n}, x_{n+1}\right)+\lambda_{2}\left(x_{0}, x_{0}\right) \frac{\left[d\left(x_{n}, x_{n+2}\right)+d\left(x_{n+1}, x_{n+1}\right)\right]}{s} \\
& +\lambda_{3}\left(x_{0}, x_{0}\right)\left[d\left(x_{n}, x_{n+1}\right)+d\left(x_{n+1}, x_{n+2}\right)\right] \\
& +\lambda_{4}\left(x_{0}, x_{0}\right) \frac{d\left(x_{n+1}, x_{n+2}\right)\left[1+d\left(x_{n}, x_{n+1}\right)\right]}{1+d\left(x_{n}, x_{n+1}\right)} \\
& +\lambda_{5}\left(x_{0}, x_{0}\right) \frac{d\left(x_{n}, x_{n+2}\right) d\left(x_{n}, x_{n+1}\right)}{s\left[1+d\left(x_{n}, x_{n+1}\right)\right]} \\
\leq & \lambda_{1}\left(x_{0}, x_{0}\right) d\left(x_{n}, x_{n+1}\right)+\lambda_{2}\left(x_{0}, x_{0}\right) \frac{d\left(x_{n}, x_{n+2}\right)}{s} \\
& +\lambda_{3}\left(x_{0}, x_{0}\right)\left[d\left(x_{n}, x_{n+1}\right)+d\left(x_{n+1}, x_{n+2}\right)\right] \\
& +\lambda_{4}\left(x_{0}, x_{0}\right) d\left(x_{n+1}, x_{n+2}\right)+\lambda_{5}\left(x_{0}, x_{0}\right) \frac{d\left(x_{n}, x_{n+2}\right)}{s} .
\end{aligned}
$$

Using triangular inequality, we get

$$
\begin{aligned}
d\left(x_{n+1}, x_{n+2}\right) \leq & \lambda_{1}\left(x_{0}, x_{0}\right) d\left(x_{n}, x_{n+1}\right)+\lambda_{2}\left(x_{0}, x_{0}\right)\left[d\left(x_{n}, x_{n+1}\right)+d\left(x_{n+1}, x_{n+2}\right)\right] \\
& +\lambda_{3}\left(x_{0}, x_{0}\right)\left[d\left(x_{n}, x_{n+1}\right)+d\left(x_{n+1}, x_{n+2}\right)\right]+\lambda_{4}\left(x_{0}, x_{0}\right) d\left(x_{n+1}, x_{n+2}\right) \\
& +\lambda_{5}\left(x_{0}, x_{0}\right)\left[d\left(x_{n}, x_{n+1}\right)+d\left(x_{n+1}, x_{n+2}\right)\right]
\end{aligned}
$$


which implies that

$$
d\left(x_{n+1}, x_{n+2}\right) \leq \frac{\sum_{i=1}^{3} \lambda_{i}\left(x_{0}, x_{0}\right)+\lambda_{5}\left(x_{0}, x_{0}\right)}{1-\sum_{i=2}^{5} \lambda_{i}\left(x_{0}, x_{0}\right)} d\left(x_{n}, x_{n+1}\right)
$$

Let $h=\frac{\sum_{i=1}^{3} \lambda_{i}\left(x_{0}, x_{0}\right)+\lambda_{5}\left(x_{0}, x_{0}\right)}{1-\sum_{i=2}^{5} \lambda_{i}\left(x_{0}, x_{0}\right)}<\frac{1}{s}$. Then

$$
d\left(x_{n+1}, x_{n+2}\right) \leq h d\left(x_{n}, x_{n+1}\right)
$$

Similarly,

$$
d\left(x_{n}, x_{n+1}\right) \leq h d\left(x_{n-1}, x_{n}\right) .
$$

Consequently,

$$
d\left(x_{n+2}, x_{n+1}\right) \leq h d\left(x_{n+1}, x_{n}\right) \leq h^{2} d\left(x_{n}, x_{n-1}\right) \leq \ldots \leq h^{n+1} d\left(x_{1}, x_{0}\right) .
$$

Now, for $m>n$ and $s h<1$, we have

$$
\begin{aligned}
d\left(x_{n}, x_{m}\right) & \leq s d\left(x_{n}, x_{n+1}\right)+s^{2} d\left(x_{n+1}, x_{n+2}\right)+\ldots+s^{m-n} d\left(x_{m-1}, x_{m}\right) \\
& \leq s h^{n} d\left(x_{1}, x_{0}\right)+s^{2} h^{n+1} d\left(x_{1}, x_{0}\right)+\ldots+s^{m-n} h^{m-1} d\left(x_{1}, x_{0}\right) \\
& \leq\left[s h^{n}+s^{2} h^{n+1}+\ldots+s^{m-n} h^{m-1}\right] d\left(x_{1}, x_{0}\right) \\
& \leq s h^{n}\left[1+(s h)^{1}+(s h)^{2}+\ldots+(s h)^{m-n-1}\right] d\left(x_{1}, x_{0}\right) \\
& \leq \frac{s h^{n}}{1-s h} d\left(x_{1}, x_{0}\right) .
\end{aligned}
$$

Therefore $\lim _{n \rightarrow \infty} d\left(x_{n}, z_{m}\right)=0$. Hence, $\left\{x_{n}\right\}$ is a cauchy sequence. But $X$ is complete, so there exists $t \in X$ such that $x_{n} \rightarrow t$ as $n \rightarrow \infty$.

Next, we show that $t$ is a fixed point of $L$. For this, assume that $L t \neq t$, then $d(t, L t) \neq 0$. Now

$$
d(t, L t) \leq d\left(t, L x_{n}\right)+d\left(L x_{n}, L t\right) .
$$

By applying condition (ii) of Theorem 2.1, equation (2.2) become

$$
\begin{aligned}
d(t, L t) \leq & d\left(t, L x_{n}\right)+\lambda_{1}\left(x_{n}, t\right) d\left(x_{n}, t\right)+\lambda_{2}\left(x_{n}, t\right) \frac{\left[d\left(x_{n}, L t\right)+d\left(t, L x_{n}\right)\right]}{s} \\
& +\lambda_{3}\left(x_{n}, t\right)\left[d\left(x_{n}, L x_{n}\right)+d(t, L t)\right]+\lambda_{4}\left(x_{n}, t\right) \frac{d(t, L t)\left[1+d\left(x_{n}, L x_{n}\right)\right]}{1+d\left(x_{n}, t\right)} \\
& +\lambda_{5}\left(x_{n}, t\right) \frac{d\left(x_{n}, L t\right) d\left(x_{n}, L x_{n}\right)}{s\left[1+d\left(x_{n}, t\right)\right]}+\lambda_{6}\left(x_{n}, t\right) \frac{d\left(x_{n}, L t\right) d\left(t, L x_{n}\right)}{s\left[1+d\left(x_{n}, t\right) d\left(t, L x_{n}\right)\right]}
\end{aligned}
$$


with the help of equation (2.1) and condition $(i)$ of Theorem 2.1, we can write

$$
\begin{aligned}
d(t, L t) \leq & d\left(t, x_{n+1}\right)+\lambda_{1}\left(x_{0}, t\right) d\left(x_{n}, t\right)+\lambda_{2}\left(x_{0}, t\right) \frac{\left[d\left(x_{n}, L t\right)+d\left(t, x_{n+1}\right)\right]}{s} \\
& +\lambda_{3}\left(x_{0}, t\right)\left[d\left(x_{n}, x_{n+1}\right)+d(t, L t)\right]+\lambda_{4}\left(x_{0}, t\right) \frac{d(t, L t)\left[1+d\left(x_{n}, x_{n+1}\right)\right]}{1+d\left(x_{n}, t\right)} \\
& +\lambda_{5}\left(x_{0}, t\right) \frac{d\left(x_{n}, L t\right) d\left(x_{n}, x_{n+1}\right)}{s\left[1+d\left(x_{n}, t\right)\right]}+\lambda_{6}\left(x_{0}, t\right) \frac{d\left(x_{n}, L t\right) d\left(t, x_{n+1}\right)}{s\left[1+d\left(x_{n}, t\right) d\left(t, x_{n+1}\right)\right]} .
\end{aligned}
$$

Taking limit as $n \rightarrow \infty$, we get

$$
\begin{gathered}
d(t, L t) \leq \lambda_{2}\left(x_{0}, t\right) \frac{d(t, L t)}{s}+\lambda_{3}\left(x_{0}, t\right) d(t, L t)+\lambda_{4}\left(x_{0}, t\right) d(t, L t) . \\
d(t, L t) \leq\left[\lambda_{2}\left(z_{0}, t\right)+s \lambda_{3}\left(z_{0}, t\right)+s \lambda_{4}\left(z_{0}, t\right)\right] \frac{d(t, L t)}{s} .
\end{gathered}
$$

But $\lambda_{2}\left(z_{0}, t\right)+s \lambda_{3}\left(z_{0}, t\right)+s \lambda_{4}\left(z_{0}, t\right)<1$, so the above inequality $(2.3)$ contradict the fact that $d(t, L t) \neq 0$. Thus $L t=t$. Hence $t$ is a fixed point of $L$.

Finally, we have to show that $t$ is a unique fixed point of $L$. For this, let $t^{*} \neq t$ be another fixed point of $L$. Then on putting $x=t$ and $y=t^{*}$ in condition $(i i)$ of Theorem 2.1, we get

$$
\begin{aligned}
d\left(t, t^{*}\right)= & d\left(L t, L t^{*}\right) \\
\leq & \lambda_{1}\left(t, t^{*}\right) d\left(t, t^{*}\right)+\lambda_{2}\left(t, t^{*}\right) \frac{\left[d\left(t, L t^{*}\right)+d\left(t^{*}, L t\right)\right]}{s} \\
& +\lambda_{3}\left(t, t^{*}\right)\left[d(t, L t)+d\left(t^{*}, L t^{*}\right)\right]+\lambda_{4}\left(t, t^{*}\right) \frac{d\left(t^{*}, L t^{*}\right)[1+d(t, L t)]}{1+d\left(t, t^{*}\right)} \\
& +\lambda_{5}\left(t, t^{*}\right) \frac{d\left(t, L t^{*}\right) d(t, L t)}{s\left[1+d\left(t, t^{*}\right)\right]}+\lambda_{6}\left(t, t^{*}\right) \frac{d\left(t, L t^{*}\right) d\left(t^{*}, L t\right)}{s\left[1+d\left(t, t^{*}\right) d\left(t^{*}, L t\right)\right]} \\
\leq & \lambda_{1}\left(t, t^{*}\right) d\left(t, t^{*}\right)+\lambda_{2}\left(t, t^{*}\right) \frac{\left[d\left(t, t^{*}\right)+d\left(t^{*}, t\right)\right]}{s}+\lambda_{6}\left(t, t^{*}\right) \frac{d\left(t, t^{*}\right) d\left(t^{*}, t\right)}{s\left[1+d\left(t, t^{*}\right) d\left(t^{*}, t\right)\right]},
\end{aligned}
$$

implies that

$$
\begin{aligned}
d\left(t, t^{*}\right) & \leq \lambda_{1}\left(t, t^{*}\right) d\left(t, t^{*}\right)+\lambda_{2}\left(t, t^{*}\right) \frac{2 d\left(t, t^{*}\right)}{s}+\lambda_{6}\left(t, t^{*}\right) \frac{d\left(t, t^{*}\right) d\left(t^{*}, t\right)}{s\left[1+d\left(t, t^{*}\right) d\left(t^{*}, t\right)\right]} \\
& \leq\left[s \lambda_{1}\left(t, t^{*}\right)+2 \lambda_{2}\left(t, t^{*}\right)+\lambda_{6}\left(t, t^{*}\right)\right] \frac{d\left(t, t^{*}\right)}{s}
\end{aligned}
$$

which is contradiction because $s \lambda_{1}\left(t, t^{*}\right)+2 \lambda_{2}\left(t, t^{*}\right)+\lambda_{6}\left(t, t^{*}\right)<1$. Hence $t$ is a unique fixed point of $L$.

From Theorem 2.1, we can easily derive the following corollaries and the proofs of which are simple, so we omit it.

Corollary 2.1. Let $(X, d)$ be a complete $b$-metric space and $\lambda_{i}: X \times X \rightarrow[0,1), i=1,3$. If $L: X \rightarrow X$ be a self-map such that for all $x, y \in X$ the following conditions are satisfied:

(i) $\lambda_{i}(L x, y) \leq \lambda_{i}(x, y)$ and $\lambda_{i}(x, L y) \leq \lambda_{i}(x, y)$ 
(ii)

$$
d(L x, L y) \leq \lambda_{3}(x, y)[d(x, L x)+d(y, L y)]
$$

where $0 \leq \lambda_{3}(x, y)<\frac{1}{s+1}$. Then the mapping $L$ has a unique fixed point in $X$.

Corollary 2.2. Let $(X, d)$ be a complete b-metric space and $\lambda_{i}: X \times X \rightarrow[0,1), i=1,2, \ldots, 8$. If $L: X \rightarrow X$ be a self-map such that for all $x, y \in X$ the following conditions are satisfied:

(i) $\lambda_{i}(L x, y) \leq \lambda_{i}(x, y)$ and $\lambda_{i}(x, L y) \leq \lambda_{i}(x, y)$;

(ii)

$$
d(L x, L y) \leq \lambda_{2}(x, y) \frac{[d(x, L y)+d(y, L x)]}{s}
$$

where $0 \leq \lambda_{2}(x, y)<\frac{1}{s+1}$. Then the mapping $L$ has a unique fixed point in $X$.

Corollary 2.3. Let $(X, d)$ be a complete b-metric space and $\lambda_{i}: X \times X \rightarrow[0,1), i=1,4$. If $L: X \rightarrow X$ be a self-map such that for all $x, y \in X$ the following conditions are satisfied:

(i) $\lambda_{i}(L x, y) \leq \lambda_{i}(x, y)$ and $\lambda_{i}(x, L y) \leq \lambda_{i}(x, y)$;

$$
d(L x, L y) \leq \lambda_{1}(x, y) d(x, y)+\lambda_{4}(x, y) \frac{d(y, L y)[1+d(x, L x)]}{1+d(x, y)},
$$

where $0 \leq s \lambda_{1}(x, y)+\lambda_{4}(x, y)<1$. Then the mapping $L$ has a unique fixed point in $X$.

Corollary 2.4. Let $(X, d)$ be a complete b-metric space and $\lambda_{i}: X \times X \rightarrow[0,1), i=1,2,3$. If $L: X \rightarrow X$ be a self-map such that for all $x, y \in X$ the following conditions are satisfied:

(i) $\lambda_{i}(L x, y) \leq \lambda_{i}(x, y)$ and $\lambda_{i}(x, L y) \leq \lambda_{i}(x, y)$;

$$
d(L x, L y) \leq \lambda_{1}(x, y) d(x, y)+\lambda_{2}(x, y) d(x, L x)+\lambda_{3}(x, y) d(y, L y)
$$

where $0 \leq s\left[\lambda_{1}(x, y)+\lambda_{2}(x, y)\right]+\lambda_{3}(x, y)<1$. Then the mapping $L$ has a unique fixed point in $X$.

Corollary 2.5. Let $\lambda_{6}=0$ and all other conditions of Theorem 2.1 are satisfied, then L has a unique fixed point in $X$.

Corollary 2.6. Let $\lambda_{5}=\lambda_{6}=0$ and all other conditions of Theorem 2.1 are satisfied, then L has a unique fixed point in $X$.

Corollary 2.7. Let $\lambda_{2}=\lambda_{3}=0$ and all other conditions of Theorem 2.1 are satisfied, then L has a unique fixed point in $X$. 
Corollary 2.8. Let $\lambda_{4}=\lambda_{5}=\lambda_{6}=0$ and all other conditions of Theorem 2.1 are satisfied, then $L$ has a unique fixed point in $X$.

\section{Remark 2.1.}

(1) In Theorem 2.1, if $s=1, \lambda_{i}=0$, for $i=2,3,4,5,6$ and $\lambda_{1}(\cdot)=\lambda_{1}$, we get the Banach Theorem [2].

(2) In Corollary 2.1, if $\lambda_{i}=0$, for $i=1,2,4,5,6, \lambda_{3}(\cdot)=\lambda$ and $s=1$, we get the Kannan Theorem [10].

(3) In Corollary 2.2, if $\lambda_{i}=0$, for $i=3,4,5,6, \lambda_{2}(\cdot)=\lambda$ and $s=1$, we get the Chatterjee Theorem [4].

(4) In Corollary 2.3, if $\lambda_{i}=0$, for $i=2,3,5,6, \lambda_{j}(\cdot)=\lambda_{j}$ for $j=1,4$ and $s=1$, we get the result of Dass and Gupta [7].

(5) In Corollary 2.4, if $s=1$ and $\lambda_{i}(\cdot)=\lambda_{i}$ for $i=1,2,3$, we get Theorem 3 of [12].

\section{Common Fixed point Results in $b$-metric spaces}

For the proof of our next result we use the following Lemma.

Lemma 3.1. Let $(X, d)$ be a complete b-metric space and $K, L: X \rightarrow X$. Let $x_{0} \in X$ and define the sequence $\left\{x_{n}\right\}$ by

$$
K x_{2 n}=x_{2 n+1} \quad \text { and } \quad L x_{2 n+1}=x_{2 n+2} \quad \forall \quad n=0,1,2, \ldots
$$

Let there exists a mapping $\lambda: X \times X \rightarrow[0,1)$ satisfying

$$
\lambda(L K x, y) \leq \lambda(x, y) \quad \text { and } \quad \lambda(x, K L y) \leq \lambda(x, y), \text { for all } \quad x, y \in X
$$

Then

$$
\lambda\left(x_{2 n}, y\right) \leq \lambda\left(x_{0}, y\right) \text { and } \lambda\left(x, x_{2 n+1}\right) \leq \lambda\left(x, x_{1}\right) \text { for all } x, y \in X
$$

Proof. The proof easily follows from lemma 2.1 .

Our next result is proved for a pair of self-maps.

Theorem 3.1. Let $(X, d)$ be a complete b-metric space with $s \geq 1$ and $\lambda_{i}: X \times X \rightarrow[0,1), i=1,2, \ldots, 5$. If $K, L: X \rightarrow X$ be two self-mappings such that for all $x, y \in X$ the following conditions are satisfied:

(i) $\lambda_{i}(L K x, y) \leq \lambda_{i}(x, y)$ and $\lambda_{i}(x, K L y) \leq \lambda_{i}(x, y)$;

$$
\begin{aligned}
d(K x, L y) \leq & \lambda_{1}(x, y) d(x, y)+\lambda_{2}(x, y) \frac{d(x, K x)[d(x, L y)+d(y, L y)]}{s[1+d(x, y)]} \\
& +\lambda_{3}(x, y) \frac{d(y, K x)[d(x, L y)+d(y, L y)]}{s[1+d(x, y)]} \\
& +\lambda_{4}(x, y) \frac{d(y, L y)[d(x, K x)+d(y, K x)]}{s[1+d(x, y)]} \\
& +\lambda_{5}(x, y) \frac{d(x, L y)[d(x, K x)+d(y, K x)]}{s[1+d(x, y)]}
\end{aligned}
$$


where $\sum_{i=2}^{5} \lambda_{i}(x, y)+s \sum_{i=1}^{5} \lambda_{i}(x, y)+\frac{1}{s}\left[\lambda_{2}(x, y)+\lambda_{4}(x, y)\right]<1$, with $0 \leq \sum_{i=1}^{5} \lambda_{i}(x, y)<1$. Then $K$ and L have a unique common fixed point in $X$.

Proof. Let $x_{0} \in X$ and construct a sequence $\left\{x_{n}\right\}$ by the rule

$$
K x_{2 n}=x_{2 n+1} \quad \text { and } \quad L x_{2 n+1}=x_{2 n+2}, \quad \forall \quad n=0,1,2, \ldots
$$

First we to show that $\left\{x_{n}\right\}$ is a Cauchy sequence in $X$. For this, consider

$$
d\left(x_{2 k+1}, x_{2 k}\right)=d\left(K L x_{2 k-1}, L x_{2 k-1}\right) .
$$

By using condition (ii) of Theorem 3.1 with $x=L x_{2 k-1}$ and $y=x_{2 k-1}$, we have

$$
\begin{aligned}
& d\left(K L x_{2 k-1}, L x_{2 k-1}\right) \\
\leq & \lambda_{1}\left(L x_{2 k-1}, x_{2 k-1}\right) d\left(L x_{2 k-1}, x_{2 k-1}\right) \\
+ & \lambda_{2}\left(L x_{2 k-1}, x_{2 k-1}\right) \frac{d\left(L x_{2 k-1}, K L x_{2 k-1}\right)\left[d\left(L x_{2 k-1}, L x_{2 k-1}\right)+d\left(x_{2 k-1}, L x_{2 k-1}\right)\right]}{s\left[1+d\left(L x_{2 k-1}, x_{2 k-1}\right)\right]} \\
+ & \lambda_{3}\left(L x_{2 k-1}, x_{2 k-1}\right) \frac{d\left(x_{2 k-1}, K L x_{2 k-1}\right)\left[d\left(L x_{2 k-1}, L x_{2 k-1}\right)+d\left(x_{2 k-1}, L x_{2 k-1}\right)\right]}{s\left[1+d\left(L x_{2 k-1}, x_{2 k-1}\right)\right]} \\
+ & \lambda_{4}\left(L x_{2 k-1}, x_{2 k-1}\right) \frac{d\left(x_{2 k-1}, L x_{2 k-1}\right)\left[d\left(L x_{2 k-1}, K L x_{2 k-1}\right)+d\left(x_{2 k-1}, K L x_{2 k-1}\right)\right]}{s\left[1+d\left(L x_{2 k-1}, x_{2 k-1}\right)\right]} \\
+ & \lambda_{5}\left(L x_{2 k-1}, x_{2 k-1}\right) \frac{d\left(L x_{2 k-1}, L x_{2 k-1}\right)\left[d\left(L x_{2 k-1}, K L x_{2 k-1}\right)+d\left(x_{2 k-1}, K L x_{2 k-1}\right)\right]}{s\left[1+d\left(L x_{2 k-1}, x_{2 k-1}\right)\right]} ;
\end{aligned}
$$

By equation (3.1), we get

$$
\begin{aligned}
d\left(x_{2 k+1}, x_{2 k}\right) \leq & \lambda_{1}\left(x_{2 k}, x_{2 k-1}\right) d\left(x_{2 k}, x_{2 k-1}\right) \\
& +\lambda_{2}\left(x_{2 k}, x_{2 k-1}\right) \frac{d\left(x_{2 k}, x_{2 k+1}\right)\left[d\left(x_{2 k}, x_{2 k}\right)+d\left(x_{2 k-1}, x_{2 k}\right)\right]}{s\left[1+d\left(x_{2 k}, x_{2 k-1}\right)\right]} \\
& +\lambda_{3}\left(x_{2 k}, x_{2 k-1}\right) \frac{d\left(x_{2 k-1}, x_{2 k+1}\right)\left[d\left(x_{2 k}, x_{2 k}\right)+d\left(x_{2 k-1}, x_{2 k}\right)\right]}{s\left[1+d\left(x_{2 k}, x_{2 k-1}\right)\right]} \\
& +\lambda_{4}\left(x_{2 k}, x_{2 k-1}\right) \frac{d\left(x_{2 k-1}, x_{2 k}\right)\left[d\left(x_{2 k}, x_{2 k+1}\right)+d\left(x_{2 k-1}, x_{2 k+1}\right)\right]}{s\left[1+d\left(x_{2 k}, x_{2 k-1}\right)\right]} \\
& +\lambda_{5}\left(x_{2 k}, x_{2 k-1}\right) \frac{d\left(x_{2 k}, x_{2 k}\right)\left[d\left(x_{2 k}, x_{2 k+1}\right)+d\left(x_{2 k-1}, x_{2 k+1}\right)\right]}{s\left[1+d\left(x_{2 k}, x_{2 k-1}\right)\right]} \\
\leq & \lambda_{1}\left(x_{2 k}, x_{2 k-1}\right) d\left(x_{2 k}, x_{2 k-1}\right)+\lambda_{2}\left(x_{2 k}, x_{2 k-1}\right) \frac{d\left(x_{2 k}, x_{2 k+1}\right) d\left(x_{2 k-1}, x_{2 k}\right)}{s\left[1+d\left(x_{2 k}, x_{2 k-1}\right)\right]} \\
& +\lambda_{3}\left(x_{2 k}, x_{2 k-1}\right) \frac{d\left(x_{2 k-1}, x_{2 k+1}\right) d\left(x_{2 k-1}, x_{2 k}\right)}{s\left[1+d\left(x_{2 k}, x_{2 k-1}\right)\right]} \\
& +\lambda_{4}\left(x_{2 k}, x_{2 k-1}\right) \frac{d\left(x_{2 k}, x_{2 k+1}\right)+d\left(x_{2 k-1}, x_{2 k+1}\right)}{s}
\end{aligned}
$$




$$
\begin{aligned}
\leq & \lambda_{1}\left(x_{2 k}, x_{2 k-1}\right) d\left(x_{2 k}, x_{2 k-1}\right)+\lambda_{2}\left(x_{2 k}, x_{2 k-1}\right) \frac{d\left(x_{2 k}, x_{2 k+1}\right)}{s} \\
& +\lambda_{3}\left(x_{2 k}, x_{2 k-1}\right) \frac{d\left(x_{2 k-1}, x_{2 k+1}\right)}{s} \\
& +\lambda_{4}\left(x_{2 k}, x_{2 k-1}\right) \frac{d\left(x_{2 k}, x_{2 k+1}\right)+d\left(x_{2 k-1}, x_{2 k+1}\right)}{s} .
\end{aligned}
$$

From Lemma 3.1 and triangular inequality, we can write

$$
\begin{aligned}
d\left(x_{2 k+1}, x_{2 k}\right) \leq & \lambda_{1}\left(x_{0}, x_{1}\right) d\left(x_{2 k}, x_{2 k-1}\right)+\lambda_{2}\left(x_{0}, x_{1}\right) \frac{d\left(x_{2 k}, x_{2 k+1}\right)}{s} \\
& +\lambda_{3}\left(x_{0}, x_{1}\right)\left[d\left(x_{2 k-1}, x_{2 k}\right)+d\left(x_{2 k}, x_{2 k+1}\right)\right]+\lambda_{4}\left(x_{0}, x_{1}\right) \frac{d\left(x_{2 k}, x_{2 k+1}\right)}{s} \\
& +\lambda_{4}\left(x_{0}, x_{1}\right)\left[d\left(x_{2 k-1}, x_{2 k}\right)+d\left(x_{2 k}, x_{2 k+1}\right)\right] .
\end{aligned}
$$

Finally one can get

$$
d\left(x_{2 k+1}, x_{2 k}\right) \leq \frac{\lambda_{1}\left(x_{0}, x_{1}\right)+\lambda_{3}\left(x_{0}, x_{1}\right)+\lambda_{4}\left(x_{0}, x_{1}\right)}{1-\left(\frac{1}{s} \lambda_{2}\left(x_{0}, x_{1}\right)+\lambda_{3}\left(x_{0}, x_{1}\right)+\frac{(1+s)}{s} \lambda_{4}\left(x_{0}, x_{1}\right)\right)} d\left(x_{2 k}, x_{2 k-1}\right)
$$

Let $h=\frac{\sum_{i=1}^{5} \lambda_{i}\left(x_{0}, x_{1}\right)}{1-\left(\frac{(1+s)}{s} \lambda_{2}\left(x_{0}, x_{1}\right)+\lambda_{3}\left(x_{0}, x_{1}\right)+\frac{(1+s)}{s} \lambda_{4}\left(x_{0}, x_{1}\right)+\lambda_{5}\left(x_{0}, x_{1}\right)\right)}<\frac{1}{s}$. Then

$$
d\left(x_{2 k+1}, x_{2 k}\right) \leq h d\left(x_{2 k}, x_{2 k-1}\right) .
$$

Similarly, consider

$$
d\left(x_{2 k-1}, x_{2 k}\right)=d\left(K x_{2 k-2}, L K x_{2 k-2}\right)
$$

By applying condition ( $i i)$ of Theorem 3.1 with $x=x_{2 k-2}$ and $y=K x_{2 k-2}$ to equation (3.3), we get

$$
\begin{aligned}
& d\left(K x_{2 k-2}, L K x_{2 k-2}\right) \\
& \leq \lambda_{1}\left(x_{2 k-2}, K x_{2 k-2}\right) d\left(x_{2 k-2}, K x_{2 k-2}\right) \\
& +\lambda_{2}\left(x_{2 k-2}, K x_{2 k-2}\right) \frac{d\left(x_{2 k-2}, K x_{2 k-2}\right)\left[d\left(x_{2 k-2}, L K x_{2 k-2}\right)+d\left(K x_{2 k-2}, L K x_{2 k-2}\right)\right]}{s\left[1+d\left(x_{2 k-2}, K x_{2 k-2}\right)\right]} \\
& +\lambda_{3}\left(x_{2 k-2}, K x_{2 k-2}\right) \frac{d\left(K x_{2 k-2}, K x_{2 k-2}\right)\left[d\left(x_{2 k-2}, L K x_{2 k-2}\right)+d\left(K x_{2 k-2}, L K x_{2 k-2}\right)\right]}{s\left[1+d\left(x_{2 k-2}, K x_{2 k-2}\right)\right]} \\
& +\lambda_{4}\left(x_{2 k-2}, K x_{2 k-2}\right) \frac{d\left(K x_{2 k-2}, L K x_{2 k-2}\right)\left[d\left(x_{2 k-2}, K x_{2 k-2}\right)+d\left(K x_{2 k-2}, K x_{2 k-2}\right)\right]}{s\left[1+d\left(x_{2 k-2}, K x_{2 k-2}\right)\right]} \\
& +\lambda_{5}\left(x_{2 k-2}, K x_{2 k-2}\right) \frac{d\left(x_{2 k-2}, L K x_{2 k-2}\right)\left[d\left(x_{2 k-2}, K x_{2 k-2}\right)+d\left(K x_{2 k-2}, K x_{2 k-2}\right)\right]}{s\left[1+d\left(x_{2 k-2}, K x_{2 k-2}\right)\right]}
\end{aligned}
$$


with the help of (3.1), we get

$$
\begin{aligned}
d\left(x_{2 k-1}, x_{2 k}\right) \leq & \lambda_{1}\left(x_{2 k-2}, x_{2 k-1}\right) d\left(x_{2 k-2}, x_{2 k-1}\right) \\
& +\lambda_{2}\left(x_{2 k-2}, x_{2 k-1}\right) \frac{d\left(x_{2 k-2}, x_{2 k-1}\right)\left[d\left(x_{2 k-2}, x_{2 k}\right)+d\left(x_{2 k-1}, x_{2 k}\right)\right]}{s\left[1+d\left(x_{2 k-2}, x_{2 k-1}\right)\right]} \\
& +\lambda_{3}\left(x_{2 k-2}, x_{2 k-1}\right) \frac{d\left(x_{2 k-1}, x_{2 k-1}\right)\left[d\left(x_{2 k-2}, x_{2 k}\right)+d\left(x_{2 k-1}, x_{2 k}\right)\right]}{s\left[1+d\left(x_{2 k-2}, x_{2 k-1}\right)\right]} \\
& +\lambda_{4}\left(x_{2 k-2}, x_{2 k-1}\right) \frac{d\left(x_{2 k-1}, x_{2 k}\right)\left[d\left(x_{2 k-2}, x_{2 k-1}\right)+d\left(x_{2 k-1}, x_{2 k-1}\right)\right]}{s\left[1+d\left(x_{2 k-2}, x_{2 k-1}\right)\right]} \\
& +\lambda_{5}\left(x_{2 k-2}, x_{2 k-1}\right) \frac{d\left(x_{2 k-2}, x_{2 k}\right)\left[d\left(x_{2 k-2}, x_{2 k-1}\right)+d\left(x_{2 k-1}, x_{2 k-1}\right)\right]}{s\left[1+d\left(x_{2 k-2}, x_{2 k-1}\right)\right]} \\
\leq & \lambda_{1}\left(x_{2 k-2}, x_{2 k-1}\right) d\left(x_{2 k-2}, x_{2 k-1}\right) \\
& +\lambda_{2}\left(x_{2 k-2}, x_{2 k-1}\right) \frac{d\left(x_{2 k-2}, x_{2 k}\right)+d\left(x_{2 k-1}, x_{2 k}\right)}{s} \\
& +\lambda_{4}\left(x_{2 k-2}, x_{2 k-1}\right) \frac{d\left(x_{2 k-1}, x_{2 k}\right)}{s}+\lambda_{5}\left(x_{2 k-2}, x_{2 k-1}\right) \frac{d\left(x_{2 k-2}, x_{2 k}\right)}{s} .
\end{aligned}
$$

Using Lemma 3.1, one can get

$$
\begin{aligned}
d\left(x_{2 k-1}, x_{2 k}\right) \leq & \lambda_{1}\left(x_{0}, x_{1}\right) d\left(x_{2 k-2}, x_{2 k-1}\right)+\lambda_{2}\left(x_{0}, x_{1}\right)\left[d\left(x_{2 k-2}, x_{2 k-1}\right)+d\left(x_{2 k-1}, x_{2 k}\right)\right] \\
& +\lambda_{2}\left(x_{0}, x_{1}\right) \frac{d\left(x_{2 k-1}, x_{2 k}\right)}{s}+\lambda_{4}\left(x_{0}, x_{1}\right) \frac{d\left(x_{2 k-1}, x_{2 k}\right)}{s} \\
& +\lambda_{5}\left(x_{0}, x_{1}\right)\left[d\left(x_{2 k-2}, x_{2 k-1}\right)+d\left(x_{2 k-1}, x_{2 k}\right)\right] .
\end{aligned}
$$

Finally,

$$
d\left(x_{2 k-1}, x_{2 k}\right) \leq \frac{\lambda_{1}\left(x_{0}, x_{1}\right)+\lambda_{2}\left(x_{0}, x_{1}\right)+\lambda_{5}\left(x_{0}, x_{1}\right)}{1-\left(\frac{(1+s)}{s} \lambda_{2}\left(x_{0}, x_{1}\right)+\frac{1}{s} \lambda_{4}\left(x_{0}, x_{1}\right)+\lambda_{5}\left(x_{0}, x_{1}\right)\right)} d\left(x_{2 k-2}, x_{2 k-1}\right)
$$

Implies that

$$
d\left(x_{2 k-1}, x_{2 k}\right) \leq h d\left(x_{2 k-2}, x_{2 k-1}\right)
$$

Now, from equations (3.2) and (3.4), we have

$$
d\left(x_{2 k+1}, x_{2 k}\right) \leq h d\left(x_{2 k}, x_{2 k-1}\right) \leq h^{2} d\left(x_{2 k-1}, x_{2 k-2}\right) .
$$

Consequently, we can write

$$
d\left(x_{n+1}, x_{n}\right) \leq h d\left(x_{n}, x_{n-1}\right) \leq h^{2} d\left(x_{n-1}, x_{n-2}\right) \leq \ldots \leq h^{n} d\left(x_{1}, x_{0}\right)
$$

Now, for $m>n$ and $s h<1$, we have 


$$
\begin{aligned}
d\left(x_{n}, x_{m}\right) & \leq s d\left(x_{n}, x_{n+1}\right)+s^{2} d\left(x_{n+1}, x_{n+2}\right)+\ldots+s^{m-n} d\left(x_{m-1}, x_{m}\right) \\
& \leq s h^{n} d\left(x_{1}, x_{0}\right)+s^{2} h^{n+1} d\left(x_{1}, x_{0}\right)+\ldots+s^{m-n} h^{m-1} d\left(x_{1}, x_{0}\right) \\
& \leq\left[s h^{n}+s^{2} h^{n+1}+\ldots+s^{m-n} h^{m-1}\right] d\left(x_{1}, x_{0}\right) \\
& \leq s h^{n}\left[1+(s h)^{1}+(s h)^{2}+\ldots+(s h)^{m-n-1}\right] d\left(x_{1}, x_{0}\right) \\
& \leq \frac{s h^{n}}{1-s h} d\left(x_{1}, x_{0}\right) .
\end{aligned}
$$

Therefore $\lim _{n \rightarrow \infty} d\left(x_{n}, z_{m}\right)=0$. Hence, $\left\{x_{n}\right\}$ is a Cauchy sequence. But $X$ is complete, so there exists $t \in X$ such that $x_{n} \rightarrow t$ as $n \rightarrow \infty$.

Next, to show that $t$ is a fixed point of $K$. For this, consider

$$
d(t, K t) \leq d\left(t, L x_{2 n+1}\right)+d\left(L x_{2 n+1}, K t\right)
$$

Using condition (ii) of Theorem 3.1 with $x=t$ and $y=x_{2 n+1}$, we have

$$
\begin{aligned}
d(t, K t) \leq & d\left(t, L x_{2 n+1}\right)+\lambda_{1}\left(t, x_{2 n+1}\right) d\left(t, x_{2 n+1}\right) \\
& +\lambda_{2}\left(t, x_{2 n+1}\right) \frac{d(t, K t)\left[d\left(t, L x_{2 n+1}\right)+d\left(x_{2 n+1}, L x_{2 n+1}\right)\right]}{s\left[1+d\left(t, x_{2 n+1}\right)\right]} \\
& +\lambda_{3}\left(t, x_{2 n+1}\right) \frac{d\left(x_{2 n+1}, K t\right)\left[d\left(t, L x_{2 n+1}\right)+d\left(x_{2 n+1}, L x_{2 n+1}\right)\right]}{s\left[1+d\left(t, x_{2 n+1}\right)\right]} \\
& +\lambda_{4}\left(t, x_{2 n+1}\right) \frac{d\left(x_{2 n+1}, L x_{2 n+1}\right)\left[d(t, K t)+d\left(x_{2 n+1}, K t\right)\right]}{s\left[1+d\left(t, x_{2 n+1}\right)\right]} \\
& +\lambda_{5}\left(t, x_{2 n+1}\right) \frac{d\left(t, L x_{2 n+1}\right)\left[d(t, K t)+d\left(x_{2 n+1}, K t\right)\right]}{s\left[1+d\left(t, x_{2 n+1}\right)\right]} .
\end{aligned}
$$

Using equation (3.1) and Proposition 3.1, we get

$$
\begin{aligned}
d(t, K t) \leq & d\left(t, x_{2 n+2}\right)+\lambda_{1}\left(t, x_{1}\right) d\left(t, x_{2 n+1}\right) \\
& +\lambda_{2}\left(t, x_{1}\right) \frac{d(t, K t)\left[d\left(t, x_{2 n+2}\right)+d\left(x_{2 n+1}, x_{2 n+2}\right)\right]}{s\left[1+d\left(t, x_{2 n+1}\right)\right]} \\
& +\lambda_{3}\left(t, x_{1}\right) \frac{d\left(x_{2 n+1}, K t\right)\left[d\left(t, x_{2 n+2}\right)+d\left(x_{2 n+1}, x_{2 n+2}\right)\right]}{s\left[1+d\left(t, x_{2 n+1}\right)\right]} \\
& +\lambda_{4}\left(t, x_{1}\right) \frac{d\left(x_{2 n+1}, x_{2 n+2}\right)\left[d(t, K t)+d\left(x_{2 n+1}, K t\right)\right]}{s\left[1+d\left(t, x_{2 n+1}\right)\right]} \\
& +\lambda_{5}\left(t, x_{1}\right) \frac{d\left(t, x_{2 n+2}\right)\left[d(t, K t)+d\left(x_{2 n+1}, K t\right)\right]}{s\left[1+d\left(t, x_{2 n+1}\right)\right]}
\end{aligned}
$$

Taking limit as $n \rightarrow \infty$, we get $d(K t, t) \leq 0$. Thus $d(K t, t)=0$ implies that $K t=t$. Hence $t$ is a fixed point of $K$.

Analogously, using condition (ii) of Theorem 3.1 with $x=x_{2 n}$ and $y=t$ one can show that $t$ is a fixed point of $L$. Therefore $K t=L t=t$, that is $t$ is a common fixed point of $K$ and $L$. 
Finally, we prove that $t$ is a unique common fixed point of $K$ and $L$. For this, suppose that $t^{*} \neq t$ be another fixed point of $K$ and $L$. Then putting $x=t$ and $y=t^{*}$ in condition (ii) of Theorem 3.1, we have

$$
\begin{aligned}
d\left(K t, L t^{*}\right) \leq & \lambda_{1}\left(t, t^{*}\right) d\left(t, t^{*}\right)+\lambda_{2}\left(t, t^{*}\right) \frac{d(t, K t)\left[d\left(t, L t^{*}\right)+d\left(t^{*}, L t^{*}\right)\right]}{s\left[1+d\left(t, t^{*}\right)\right]} \\
& +\lambda_{3}\left(t, t^{*}\right) \frac{d\left(t^{*}, K t\right)\left[d\left(t, L t^{*}\right)+d\left(t^{*}, L t^{*}\right)\right]}{s\left[1+d\left(t, t^{*}\right)\right]} \\
& +\lambda_{4}\left(t, t^{*}\right) \frac{d\left(t^{*}, L t^{*}\right)\left[d(t, K t)+d\left(t^{*}, K t\right)\right]}{s\left[1+d\left(t, t^{*}\right)\right]} \\
& +\lambda_{5}\left(t, t^{*}\right) \frac{d\left(t, L t^{*}\right)\left[d(t, K t)+d\left(t^{*}, K t\right)\right]}{s\left[1+d\left(t, t^{*}\right)\right]}
\end{aligned}
$$

which implies that

$$
\begin{aligned}
d\left(t, t^{*}\right) & \leq \lambda_{1}\left(t, t^{*}\right) d\left(t, t^{*}\right)+\lambda_{3}\left(t, t^{*}\right) \frac{d\left(t^{*}, t\right) d\left(t, t^{*}\right)}{s\left[1+d\left(t, t^{*}\right)\right]}+\lambda_{5}\left(t, t^{*}\right) \frac{d\left(t, t^{*}\right) d\left(t^{*}, t\right)}{s\left[1+d\left(t, t^{*}\right)\right]} \\
& \leq \lambda_{1}\left(t, t^{*}\right) d\left(t, t^{*}\right)+\lambda_{3}\left(t, t^{*}\right) \frac{d\left(t^{*}, t\right)}{s}+\lambda_{5}\left(t, t^{*}\right) \frac{d\left(t, t^{*}\right)}{s} \\
& \leq\left[s \lambda_{1}\left(t, t^{*}\right)+\lambda_{3}\left(t, t^{*}\right)+\lambda_{5}\left(t, t^{*}\right)\right] \frac{d\left(t, t^{*}\right)}{s} .
\end{aligned}
$$

Which is contradiction because $s \lambda_{1}\left(t, t^{*}\right)+\lambda_{3}\left(t, t^{*}\right)+\lambda_{5}\left(t, t^{*}\right)<1$, thus $d\left(t^{*}, t\right)=0$ and hence $t^{*}=t$. Therefore $t$ is a unique common fixed point of $K$ and $L$.

From Theorem 3.1, we can derive the following corollaries and the proof of which is simple, so we omit it.

Corollary 3.1. If $K=L$ and all other conditions of Theorem 3.1 are satisfied, then $L$ has a unique fixed point in $X$.

Corollary 3.2. Let $(X, d)$ be a complete b-metric space with $s \geq 1$ and $\lambda_{i}: X \times X \rightarrow[0,1), i=1,2$. If $K, L: X \rightarrow X$ be two self-mappings such that for all $x, y \in X$ the following conditions are satisfied:

(i) $\lambda_{i}(L K x, y) \leq \lambda_{i}(x, y)$ and $\lambda_{i}(x, K L y) \leq \lambda_{i}(x, y)$;

$$
d(K x, L y) \leq \lambda_{1}(x, y) d(x, y)+\lambda_{2}(x, y) \frac{d(x, K x)[d(x, L y)+d(y, L y)]}{s[1+d(x, y)]}
$$

where $0 \leq s \lambda_{1}(x, y)+\left(\frac{s^{2}+s+1}{S}\right) \lambda_{2}(x, y)<1$. Then $K$ and $L$ have a unique common fixed point in $X$.

Corollary 3.3. Let $(X, d)$ be a complete b-metric space with $s \geq 1$ and $\lambda_{i}: X \times X \rightarrow[0,1), i=1,4$. If $K, L: X \rightarrow X$ be two self-mappings such that for all $x, y \in X$ the following conditions are satisfied:

(i) $\lambda_{i}(L K x, y) \leq \lambda_{i}(x, y)$ and $\lambda_{i}(x, K L y) \leq \lambda_{i}(x, y)$;

$$
d(K x, L y) \leq \lambda_{1}(x, y) d(x, y)+\lambda_{4}(x, y) \frac{d(y, L y)[d(x, K x)+d(y, K x)]}{s[1+d(x, y)]}
$$


where $0 \leq s \lambda_{1}(x, y)+\left(\frac{s^{2}+s+1}{S}\right) \lambda_{4}(x, y)<1$. Then $K$ and L have a unique common fixed point in $X$.

Corollary 3.4. Let $(X, d)$ be a complete $b$-metric space with $s \geq 1$ and $\lambda_{i}: X \times X \rightarrow[0,1), i=1,2,4$. If $K, L: X \rightarrow X$ be two self-mappings such that for all $x, y \in X$ the following conditions are satisfied:

(i) $\lambda_{i}(L K x, y) \leq \lambda_{i}(x, y)$ and $\lambda_{i}(x, K L y) \leq \lambda_{i}(x, y)$

$$
\begin{aligned}
d(K x, L y) \leq & \lambda_{1}(x, y) d(x, y)+\lambda_{2}(x, y) \frac{d(x, K x)[d(x, L y)+d(y, L y)]}{s[1+d(x, y)]} \\
& +\lambda_{4}(x, y) \frac{d(y, L y)[d(x, K x)+d(y, K x)]}{s[1+d(x, y)]}
\end{aligned}
$$

where $0 \leq s \lambda_{1}(x, y)+\left(\frac{s^{2}+1}{S}\right) \lambda_{2}(x, y)+\left(\frac{s^{2}+s+1}{S}\right) \lambda_{4}(x, y)<1$. Then $K$ and L have a unique common fixed point in $X$.

For the validity of Theorem 3.1, we construct the following example.

Example 3.1. Let $X=[0,1]$ and $d: X \times X \rightarrow \mathbb{R}^{+}$defined by $d(x, y)=(\alpha|x-y|)^{2}=\alpha^{2}|x-y|^{2}$ with $\alpha \geq 8, s=2$. Define $K, L: X \rightarrow X$ by $K x=\frac{x}{4}$ and $L x=\frac{x}{5}$. Let $\lambda_{i}: X \times X \rightarrow[0,1), i=1,2, \ldots, 5$ are defined as:

$$
\begin{aligned}
& \lambda_{1}(x, y)=\frac{x}{19}+\frac{y}{21}, \quad \lambda_{2}(x, y)=\frac{x}{17}+\frac{y}{23}, \quad \lambda_{3}(x, y)=\frac{x^{2}}{29}+\frac{y^{2}}{37}, \quad \lambda_{4}(x, y)=\frac{x^{3}+y^{3}}{41} \\
& \lambda_{5}(x, y)=\frac{x y}{43}
\end{aligned}
$$

To check condition $(i)$, we have, since $L K x=\frac{x}{20}$ and $K L y=\frac{y}{20}$. Then by routine calculation, one can easily obtained that

$$
\lambda_{i}(L K x, y) \leq \lambda_{i}(x, y) \text { and } \lambda_{i}(x, K L y) \leq \lambda_{i}(x, y) \text { for all } i=1,2, \ldots, 5
$$

To check condition (ii), we have,

$$
\begin{aligned}
d(K x, L y)= & \alpha^{2}|K x-L y|^{2}=\alpha^{2}\left|\frac{x}{4}-\frac{y}{5}\right|^{2} \\
\leq & \left(\frac{x}{19}+\frac{y}{21}\right) \alpha^{2}|x-y|^{2}+\left(\frac{x}{17}+\frac{y}{23}\right) \frac{\alpha^{4}\left|x-\frac{x}{4}\right|^{2}\left[\left|x-\frac{y}{5}\right|^{2}+\left|y-\frac{y}{5}\right|^{2}\right]}{2\left[1+\alpha^{2}|x-y|^{2}\right]} \\
& +\left(\frac{x}{29}+\frac{y}{37}\right) \frac{\alpha^{4}\left|y-\frac{x}{4}\right|^{2}\left[\left|x-\frac{y}{5}\right|^{2}+\left|y-\frac{y}{5}\right|^{2}\right]}{2\left[1+\alpha^{2}|x-y|^{2}\right]} \\
& +\left(\frac{x^{3}+y^{3}}{41}\right) \frac{\alpha^{4}\left|y-\frac{y}{5}\right|^{2}\left[\left|x-\frac{x}{4}\right|^{2}+\left|y-\frac{x}{4}\right|^{2}\right]}{2\left[1+\alpha^{2}|x-y|^{2}\right]} \\
& +\left(\frac{x y}{43}\right) \frac{\alpha^{4}\left|x-\frac{y}{5}\right|^{2}\left[\left|x-\frac{x}{4}\right|^{2}+\left|y-\frac{x}{4}\right|^{2}\right]}{2\left[1+\alpha^{2}|x-y|^{2}\right]}
\end{aligned}
$$




$$
\begin{aligned}
d(K x, L y)= & \lambda_{1}(x, y) d(x, y)+\lambda_{2}(x, y) \frac{d(x, K x)[d(x, L y)+d(y, L y)]}{s[1+d(x, y)]} \\
& +\lambda_{3}(x, y) \frac{d(y, K x)[d(x, L y)+d(y, L y)]}{s[1+d(x, y)]} \\
& +\lambda_{4}(x, y) \frac{d(y, L y)[d(x, K x)+d(y, K x)]}{s[1+d(x, y)]} \\
& +\lambda_{5}(x, y) \frac{d(x, L y)[d(x, K x)+d(y, K x)]}{s[1+d(x, y)]}
\end{aligned}
$$

where $\sum_{i=2}^{5} \lambda_{i}(x, y)+2 \sum_{i=1}^{5} \lambda_{i}(x, y)+\frac{1}{2}\left[\lambda_{2}(x, y)+\lambda_{4}(x, y)\right]<1$.

Thus all the conditions of Theorem 3.1 are satisfied, hence $K$ and $L$ has a unique fixed point $0 \in X$.

To state the next result, we need the following Lemma the proof of which can be easily obtained from Lemma 2.1.

Lemma 3.2. Let $(X, d)$ be a complete $b$-metric space with $s \geq 1$ and $K, L: X \rightarrow X$. Let $x_{0} \in X$ and define the sequence $\left\{x_{n}\right\}$ by

$$
K x_{2 n}=x_{2 n+1} \quad \text { and } \quad L x_{2 n+1}=x_{2 n+2} \quad \forall \quad n=0,1,2, \ldots
$$

Assume that there exists a mapping $\lambda: X \rightarrow[0,1)$ such that $\lambda(L K x) \leq \lambda(x)$, for all $x \in X$. Then $\lambda\left(x_{2 n}\right) \leq$ $\lambda\left(x_{0}\right)$ and $\lambda\left(x_{2 n+1}\right) \leq \lambda\left(x_{1}\right)$ for all $x \in X$ and $n=0,1,2, \ldots$

Theorem 3.2. Let $(X, d)$ be a complete $b$-metric space with $s \geq 1$ and $\lambda_{i}: X \rightarrow[0,1), i=1,2, \ldots, 9$. If $K, L: X \rightarrow X$ be two self-mappings such that for all $x, y \in X$ the following conditions are satisfied:

(i) $\lambda_{i}(L K x) \leq \lambda_{i}(x)$

(ii)

$$
\begin{aligned}
d(K x, L y) \leq & \lambda_{1}(x) d(x)+\lambda_{2}(x) \frac{d(x, K x)[d(x, L y)+d(y, L y)]}{s[1+d(x, y)]} \\
& +\lambda_{3}(x) \frac{d(y, K x)[d(x, L y)+d(y, L y)]}{s[1+d(x, y)]}+\lambda_{4}(x) \frac{d(y, L y)[d(x, K x)+d(y, K x)]}{s[1+d(x, y)]} \\
& +\lambda_{5}(x) \frac{d(x, L y)[d(x, K x)+d(y, K x)]}{s[1+d(x, y)]}
\end{aligned}
$$

where $\sum_{i=2}^{5} \lambda_{i}(x)+s \sum_{i=1}^{5} \lambda_{i}(x)+\frac{1}{s}\left[\lambda_{2}(x)+\lambda_{4}(x)\right]<1$, with $0 \leq \sum_{i=1}^{5} \lambda_{i}(x)<1$. Then $K$ and L have a unique common fixed point in $X$.

Proof. By using Lemma 2.1 and following the same steps as in Theorem 3.1 one can easily prove the Theorem.

One can deduce corollaries from Theorem 3.2 in the same way as derived from Theorem 3.1. 


\section{ACKNOWLEDGEMENTS}

The authors wish to thank the editor and anonymous referees for their comments and suggestions, which helped to improve this paper.

\section{REFERENCES}

[1] I. A. Bakhtin, The contraction mapping principle in quasimetric spaces, Funct. Anal., Unianowsk Gos. Ped. Inst. 30(1989), $26-37$.

[2] S. Banach, Sur les oprations dans les ensembles abstraits et leurs applications aux equations integrales, Fund. Math. 3(1922), 133-181.

[3] M. Boriceanu, M. Bota, A. Petrusel, Multivalued fractals in $b-$ metric spaces, Cent. Eur. J. Math. 8(2010), 367-377.

[4] S. K. Chetterjea, Fixed Point Theorems, C. R. Acad. Bulgara Sci. 25(1972), 727-730.

[5] S. Czerwik, Contraction mappings in b-metric spaces, Acta Math. Inform. Univ. Ostrav. 1(1993), 5-11.

[6] S. Czerwik, Nonlinear set-valued contraction mappings in $b$-metric spaces, Atti Sem. Mat. Fis. Univ. Modena, 46(1998), $263-276$.

[7] B. K. Dass, S. Gupta, An extension of Banach contraction principle through rational expression, Indian J. Pure Appl. Math. 6(1975), 1455-1458.

[8] N. Hussain, V. Parvaneh, J. R. Roshan, Z. Kadelburg, Fixed points of cyclic weakly $(\psi, \phi, L, A, B)$-contractive mappings in ordered b-metric spaces with applications, Fixed Point Theory Appl. 2013 (2013), Art. ID 256.

[9] N. Hussain, D. Dorić, Z. Kadelburg, S. Radenović, Suzuki-type fixed point results in metric type spaces, Fixed Point Theory Appl. (2012)2012, Art. ID 126.

[10] R. Kannan, Some results on fixed points, Bull. Calcutta Math. Soc. 6(1968), 71-78.

[11] P. Kumam, N. V. Dung, V. T. Le Hang, Some equivalences between cone $b$-metric spaces and b-metric spaces, Abstr. Appl. Anal. 2013(2013), Art. ID 573740.

[12] S. Reich, Some remarks concerning contraction mappings, Canad. Math. Bull. 14(1971), 121-124.

[13] S. L. Singh, B. Prasad, Some coincidence theorems and stability of iterative procedures, Comput. Math. Appl. 55(2008), $2512-2520$.

[14] W. Sintunavarat, Nonlinear integral equations with new admissibility types in $b$-metric spaces, J. Fixed Point Theory Appl. 18(2016), 397416. 